\title{
On Reacting to Assertions and Polar Questions
}

\author{
Donka F. Farkas \\ UC Santa Cruz \\ Kim B. Bruce \\ Pomona College
}

July 21,2009

\begin{abstract}
$^{1}$
The aim of this paper is to capture the similarities and differences between assertions and polar questions so as to be able to account for the systematic partial overlap that exists in reactions to these speech acts in English and beyond. We first discuss the discourse components we assume, and then define default assertions and default polar questions in a way that allows us to characterize two types of responses to these speech acts, confirming and reversing reactions. The common characteristics of assertions and polar questions are responsible for the fact that both allow these reactions; the differences between the two speech acts explain the different contextual effects confirming and reversing moves have depending on whether they react to an assertion or a polar question. We then examine the distribution of a set of 'polarity' particles in Romanian in terms of the notions defined in the rest of the paper, and end with a series of predictions concerning polarity particles across languages.
\end{abstract}

\section{Introduction}

Our aim here is to explain the systematic similarities and contrasts between a particular class of reactions to assertions and polar questions. We start, in Section 2, by laying out the context structure we use in the rest of the paper, and then turn in Section 3 to a characterization of assertions and polar questions against this background. In Section 4 we explore a special type of reactions to assertions and polar questions that exhibits the partial overlap we are interested in capturing. In Section 5 we study the distribution of a set of particles occuring in a subtype of such reactions, and then conclude. In the rest of this section we provide an introduction to the issues the paper engages with.

One of the cornerstones of work on discourse structure is Stalnaker (1978), where it is suggested that discourse unfolds against an ever changing background made up of a set of propositions already confirmed by the discourse participants, the common ground $(\mathrm{cg})$, whose intersection is the context set $(\mathrm{cs})$. In what follows we adopt this view of the $c g^{2}$

The essential effect of assertions on the context in which they are made, according to Stalnaker, is to add the content of the assertion to the $c g$, 'provided', Stalnaker adds, 'that there are no objections from the other participants in the conversation.' Assertion, in this view, is a proposal to change the context by adding the

\footnotetext{
${ }^{1}$ We thank audiences at talks given at UCSC and Pomona College for useful questions and comments. We are also grateful to Bart Geurts and three anonymous referees for detailed, careful and useful suggestions. All remaining errors are, of course, our own.

${ }^{2}$ Note that Stalnaker's $c g$ corresponds to grounding at level 4 in Clark (1992), Clark \& Schaefer (1989). For recent discussion of matters pertaining to grounding at lower levels, see Ginzburg (forthcoming).
} 
propositional content of the asserted sentence to the $c g .{ }^{3}$ We follow Clark (1992); Clark \& Schaefer (1989) and Ginzburg (1996, forthcoming), among many others, in emphasizing the proposal nature of assertion, an aspect that Stalnaker himself does not stress. Characterizing ordinary assertion as proposing additions to the $c g$, rather than actually changing it, is necessary in order to make room for the large variety of conversational moves that react to assertions signaling, for instance, confirmation, rejection or the need for further discussion, as exemplified in (1):

Anne: Sam's home.

Ben: Yeah, he is./No, he isn't./ Why do you think so?

Earlier, as well as very recent work (see, for instance Hamblin (1971) and Gunlogson (2001)), has stressed the role of public individual commitments in discourse. According to Gunlogson (2001), the main effect of uttering a declarative sentence with falling intonation is to add its propositional content to the speaker's list of public commitments. This view, we claim, goes too far in neglecting the fact that such speech acts also steer the conversation towards turning the content of the declarative sentence into a shared, or joint commitment. The context components we adopt here allow us to capture both the commitment publicizing and the proposal nature of default assertions. As we will see below, in contrast with assertions, default polar questions do not register author commitment. They propose to add to the $c g$ either the denotation of their sentence radical or its complement.

The fact that assertions project the addition of a proposition to the $c g$ is crucial to the empirical focus of our work, namely capturing the similarities and differences between reactions to assertions and reactions to polar questions. This is an issue that has not received the attention it deserves. The overlap between the two types of reactions is illustrated in (2):

Anne: Sam is home.

Ben: Yes/Yeah, he's home./No, he isn't home.

Anne: Is Sam home?

Ben: Yes/Yeah, he's home./No, he isn't home.

On the differences side, we argue that Ben's negative reaction in (2) creates a conversational crisis while his negative reaction in (3) does not. Anne's contributions in (2) and (3) raise the issue of Sam's whereabouts; in (2), but not (3), she also adds the proposition denoted by Sam is home to her public commitments.

At the root of the 'conversational crisis' brought about by Ben's second reaction in (2) are the following two assumptions: (i) conversational changes that result in additions to commitments are less marked than those that result in the retraction of such commitments, and (ii) raising an issue steers the conversation toward a state where the issue is resolved. Now in (3) Anne can go on to accept either one of Ben's responses without further ado thus reaching a conversational state in which the issue is resolved by the addition of a proposition to the $c g$. In (2), on the other hand, after Ben's negative reaction the issue of Sam's whereabouts can be settled only after either Ben or Anne retracts a commitment. Ben's negative reaction in (3) then is more dramatic than in (2) because it takes the conversation to a state from which reaching the canonical future of settling the issue raised by Anne can be reached only via a non-canonical move of commitment retraction.

The difference between the contextual effect of contradicting an assertion and giving the 'opposite'

\footnotetext{
${ }^{3}$ A large amount of work since Stalnaker (1978) has been devoted to the need to refine the notion of context so as to get to the level of discourse referents. The issues we are dealing with here are not relevant to these concerns and therefore they will be ignored below.
} 
answer to a polar question affects their formal properties as well. We illustrate the difference with Romanian, where the particle $b a$ is possible when contradicting a positive assertion but not in a negative answer to a positive question:

Ana: Horea e acasă? 'Is Horea home?'

Petru: Nu, nu e./*Ba nu, nu e. 'No, he isn't.'

Ana: Horea e acasă. 'Horea is home.'

Petru: Nu, nu e./Ba nu, nu e. 'No, he isn't.'

We return to these particles in Section $5 .{ }^{4}$

We have briefly illustrated here that there are significant parallelisms between reactions to assertions and reactions to polar questions but that the two diverge as well. Our main goal in this paper is to capture this partial overlap. While reaching it we also have to differentiate between initiating assertions (exemplified by Anne's conversational contributions above) and 'reactive' assertions (exemplified by Ben's contributions) in a way that allows us to account for the distribution of particles such as yes and no and their relatives in other languages. But first we turn in the next sextion to the context components we work with.

\section{Context components}

In this section we present a model of context structure that combines features of earlier proposals, especially those in Ginzburg (1996), Ginzburg (forthcoming), Gunlogson (2001) and Asher \& Lascarides (2003), within the tradition of foundational work by Stalnaker (1978), Hamblin (1971), and Carlson (1983). Since our focus is to capture the similarities and differences between assertions and polar questions, we do not go beyond those elements of context structure that are necessary for our task.

An essential component of models of context, acknowledged since the earliest works on the topic, is the representation of the discourse commitments of participants. We assume here that discourse commitments are propositions (i.e., sets of worlds) publicly taken by the participants in a conversation as being true of the world of the conversation, from the perspective of the conversation. (See, for instance, Sacks (1992). $)^{5}$

A much debated issue is how to relate discourse commitments to participants. Stalnaker (1978) assumes a single set of shared public commitments, the $\mathrm{cg}$. In Hamblin (1971) and Gunlogson (2001), on the other hand, each participant is associated with a set of discourse commitments. Gunlogson (2001) defines the common ground as an ancillary notion made up of the union of the participants' commitment sets. Stalnaker's $c g$ can be defined as the intersection of these sets. In Ginzburg's work there is a common ground, called FACTS, as well as a dialogue game board containing both public and private aspects associated with each participant in the conversation. In Asher \& Lascarides (2003), each participant is associated with a Segmented Discourse Representation Structure (SDRS) that represents their cognitive state. The common ground in Asher and Lascarides is reconstructed via a relation, called settled, defined between a content and a discourse structure. ${ }^{6}$

\footnotetext{
${ }^{4}$ As noted by an anonymous reviewer, a further difference between default assertions and default polar questions is that reactions such as You are right/You are wrong and their equivalents in other languages are possible after the former but not the latter. Such reactions can only follow moves that add a commitment to their author's list, which assertions do but default polar questions do not.

${ }^{5}$ Note also that in the sense in which we use the term discourse commitment, participants can only be committed to propositions and not to 'issues' or 'questions'.

${ }^{6}$ This relation is more general than the Stalnakerian $c g$ because 'settled' contents include not only agreed upon propositions, but also questions participants are committed to supplying an answer to as well as requests they are publicly committed to carrying out. For recent refinements of Asher and Lascarides' view see Asher \& Lascarides (2008) and Lascarides \& Asher (2008).
} 
We present here a model that separates a Stalnakerian $c g$ from the discourse commitment set of each participant. The discourse commitment set of a participant $\mathrm{A}$ at a time $t$ in a conversation $c$ contains those propositions A has publicly committed to in the course of $c$ up to $t$ and which have not (yet) become mutual commitments. The $c g$ on the other hand, is that set of propositions that have been agreed upon by all participants in $c$ at $t$ together with the propositions that represent the shared background knowledge of the discourse participants.

Having the $c g$ as a separate component allows us to capture what Stalnaker takes the crucial effect of assertions to be, namely that of proposing to add the propositional content of the asserted sentence to the $c g$. This aspect of ordinary assertive speech acts allows us to explain the fact that assertion acceptance is a default move while assertion contradiction or denial is not. It also allows us to explain the existence of conversational pressure in the direction of increasing the $\mathrm{cg}$ by turning publicized commitments into joint commitments. Without such pressure the Collaborative Principle of Walker (1996) would remain mysterious. This principle requires participants to provide evidence of detected 'discrepancy in belief' as soon as possible in a conversation. Were it not for the drive to build a $c g$, discrepancy in publicized belief would not be problematic, and thus in no special need of being signaled.

Commitment sets (whether the $c g$ or individual commitment sets) can be upgraded or downgraded. The former change, we take it, is more canonical than the latter. See again Walker (1996), who assumes the existence of a default rule that makes beliefs persistent. In our terms, this means that addition is a canonical change to a commitment set while retraction is not.

More concretely, we assume that a discourse structure $K$ contains a (possibly empty) set of propositions $D C_{X}$ for each participant $X$ in the conversation, made up of those propositions that $X$ has publicly committed to during the conversation up to the relevant time, and which are not shared by all the other participants. In addition, $K$ contains a set of propositions, the $c g$, whose elements are those propositions that have been confirmed by all participants in the conversation as well as a set of background propositions. The total discourse commitments of a discourse participant $X$ is $D C_{X} \cup \mathrm{cg}$. By default, the set of total discourse commitments of a participant is taken to be a subset of her doxastic base (cf. Grice's Quality Maxim). Discourse commitments are not required to be correct, and therefore they need not be in fact true of the world in which the conversation takes place, but from the perspective of the conversation, they are assumed to be correct. Because of this assumption, a discourse participant is coherent iff her total discourse commitments are consistent and a discourse whose $c g$ is inconsistent is in crisis.

Separating participants' discourse commitments from the $c g$ is essential in accounting for disagreements in ordinary discourses. One way a discourse may survive denial, for instance, is when participants agree to disagree. Leaving the details for Section 4, assume now that A has committed to $p$ during a conversation with $\mathrm{B}$, while $\mathrm{B}$ has committed to $\neg p$, and that $\mathrm{A}$ and $\mathrm{B}$ have agreed to disagree on $p$. The state of the discourse in this case should record A's commitment to $p$ and B's commitment to $\neg p$ but neither $p$ nor $\neg p$ should be in the $c g$. In our model, in such a case $p$ will be an element of $D C_{A}, \neg p$ will be an element of $D C_{B}$ and neither $p$ nor $\neg p$ will be an element of the $c g$. Thus, it is possible to have a conversation state with a consistent $c g$ and coherent participants even though $D C_{A} \cup D C_{B}$ is not consistent. This, we take it, is a welcome result. Note also that the level that counts for purposes of presupposition satisfaction in such cases is the $c g$ rather than either of the participant's commitment set. Thus, after having agreed to disagree on whether Sam is home, it would be infelicitous for A to say Since Sam is home, the children are not alone even if A has previously committed to Sam being home.

We follow the literature, and especially Ginzburg (1996), Roberts (1996), and Büring (2003), in having a discourse component that records the questions under discussion (Ginzburg's QUD). We rechristen it the Table and assume that the items on it are syntactic objects paired with their denotations. Having syntactic 
objects on the Table is needed because a grammar of cross-turn conversation and ellipsis has to have access to the grammatical form (and not just the content) of immediately previous utterances. For instance, in many languages polar questions can be answered by repeating the main verb of the question with the same or the reverse polarity. Access to the morpho-syntax of the question is needed to account for the form of these 'fragment answers' independently of the finer details of their treatment. Given what was just said, asserting a sentence such as Sam is home makes a different contribution to the Table from asking the question Is Sam home?, since the former adds a declarative sentence while the latter adds an interrogative one.

For simplicity we assume that the items on the Table form a stack. ${ }^{7}$ The Table records what is 'at issue' in the conversation. When the Table is not empty, the immediate goal of the conversation is to empty it, i.e. to settle the issue at hand. The stack discipline allows us to capture the tight connection between initiating conversational moves, such as an initiating assertion or question, and responding moves that address the issue an immediately preceding utterance has placed on the Table, a matter we come back to in Section 4.

Finally, having the Table as a separate component allows us to differentiate between context states that can serve as natural endpoints of a discourse, and states that cannot. As long as there are items on the Table, there are issues that need to be dealt with. A conversation is in a stable state when its Table is empty; a conversation is at a natural endpoint only when it is stable.

We assume that two fundamental engines that drive conversations are the need to increase the $c g$ and the need to reach a stable state. The former drives participants to place items on the Table; the latter drives them to take the necessary steps to remove these items in such a way as to increase the $\mathrm{cg}$. A novelty of our approach is that we attempt to capture these twin goals by assuming that every conversational move that places an item on the Table is associated with a canonical way of removing that item from the Table. The canonical way of removing an issue from the Table is to reach a discourse state in which the issue is decided. A proposition $p$ is decided relative to a common ground $c g$ if and only if $p$ follows from the $c g$ or if $\neg p$ follows from the $c g$. If a proposition $p$ is decided relative to the common ground $c g_{K}$ of a conversational state $K, p$ is decided in $K .^{8}$

More specifically, we assume that a conversational move that places an item on the Table simultaneously projects a set of future common grounds relative to which the issue on the Table is decided. These projected sets are supersets of the current $c g$. We call these privileged future common grounds the projected set ( $p s$ ) and represent them in a separate component. As we see in detail in the next section, an assertion projects confirmation in that it projects a future common ground that includes the asserted proposition. A question projects resolution in the sense that it projects a set of future common grounds arrived at by adding to each common ground in the input $p s$ each (contextually) possible answer to the question. Assertions and questions are similar in that their effect on the $p s$ is to add their denotations to the elements of the input ps. They differ in that for assertions, only one proposition is added and therefore only one resolution is projected, while in the case of questions one adds a non-singleton set of propositions, thus projecting a non-singleton set of resolutions. When the Table is empty, the $p s$ contains the current $c g$ as its only element. When the Table is not empty, the elements of the $p s$ are computed based on what is on the Table. ${ }^{9}$

\footnotetext{
${ }^{7}$ Ginzburg (1995, forthcoming) prefers a partial order, but we have no need for the extra flexibility in the simple examples handled here.

${ }^{8}$ In order to render these definition precise one needs to give an underlying logic and semantics that defines when a proposition follows from a set of propositions. Simply assuming that a proposition follows from a set of propositions if the intersection of the latter is a subset of the former is too course-grained for our purposes since in that case all logically necessary propositions would count as decided in all conversations. In this paper we focus on the context structures needed to represent discourse states and simply assume that such an underlying logic and semantics exists. What we propose here is consistent with the logic in Asher \& Lascarides (2003) and Asher \& Lascarides (2008).

${ }^{9}$ Our ps is different from the projection set of Gunlogson (2001), which contains all non-empty subsets of the current context set.
} 
The ps assumes a central role in our view because it is at this level that the anticipatory nature of certain conversational moves is captured. In this respect we differ from most previous work where the fact that moves may steer conversations towards particular privileged futures is not directly represented in the conversational state. Thus, in Asher and Lascarides's work, for instance, a move affects the future of the conversation only in so far as future moves must find a possible rhetorical relation that attaches them to it. This is different from but compatible with our proposal. We distinguish privileged future states that particular conversational moves direct the conversation toward. An assertion may be contradicted, confirmed, called into question, elaborated on or corrected. Out of all possible reactions, we capture the special nature of confirmation by assuming that this is what is projected at the $p s$ level. Similarly, resolution is only one of the many possible ways in which one can react to a question. We treat resolution as the privileged future projected by raising the question.

Thus, in our view, moves that place items on the Table intrinsically define a conversational goal state (or set of such states) reached when the item in question is removed from the Table in a way that maximally increases the $c g .{ }^{10}$ We use the $p s$ in characterizing what A's proposal is when she makes her assertion, as well as what the questioner directs the conversation toward when he asks his question. Furthermore, the $p s$ allows us to import the insights of earlier work on the context change of assertions and questions, but, instead of performing these changes at the level of the $\mathrm{cg}$ (or its intersection, the context set), we perform them at the level of the $p s$.

We take it that a conversation is in crisis if its $c g$ is inconsistent or if all the sets in its $p s$ are inconsistent. Requiring consistency of the $c g$ is a consequence of the assumption that the propositions in the $c g$ are assumed, for the purposes of the conversation, to be true of the world in which the conversation takes place. Having at least one consistent set in the $p s$ means that there are canonical moves that lead to emptying the Table while at the same time reaching a consistent $c g$.

For the matters we discuss here, further additions to context structure such as the agendas of participants or representations of their private doxastic states are not necessary. The model we provide is consistent with expansion in these directions, as well as with additions of finer grained structures for dealing with anaphoric relations.

Finally, we note that the information we do represent is redundant for the examples we discuss. In particular, the contents of the $p s$ can always be calculated from the current $c g$ and the items on the Table. We nevertheless include it in our representation for the sake of clarity and for emphasizing similarities between what we propose and previous approaches.

We represent the context $K$ of a conversation between two participants $\mathrm{A}$ and $\mathrm{B}$ in diagrams of the form below:

Sample context structure

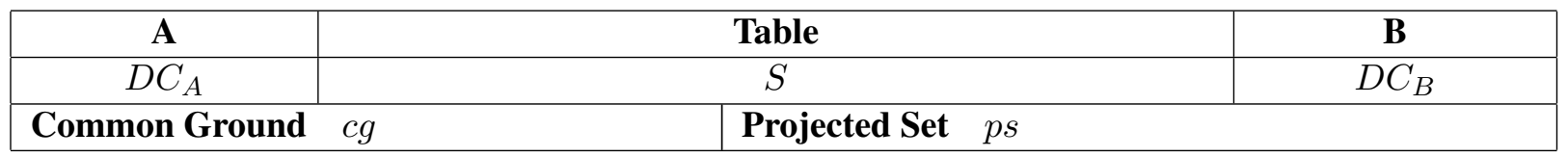

Here $D C_{A}$ and $D C_{B}$ are the propositions $\mathrm{A}$ and $\mathrm{B}$ have individually publicly committed to and which are not joint commitments. The $c g$ is the set of propositions that are joint commitments of the participants. The contents of the Table, $S$, are items that are still under discussion, while the $p s$ is a set of common grounds

\footnotetext{
${ }^{10}$ Our canonical Table-emptying operations correspond to Ginzburg's operations that downgrade QUD and upgrade the FACTS once a resolution of the issue on the Table has been agreed upon.
} 
that reflects canonical ways of settling the issues on the Table.

Before turning to discussing assertions and polar questions, we define here the notation we use throughout the rest of the paper. The table $T$ in a context structure is represented as a stack. The following notation is used to represent traditional stack operations on $T$ :

1. $\operatorname{push}(e, T)$ represents the new stack obtained by adding item $e$ to the top of the stack $T$.

2. $\operatorname{pop}(T)$ represents the stack obtained by popping off the top item of $T$.

3. $\operatorname{top}(T)$ represents the top item of the stack $T$.

4. remove $(e, T)$ represents the stack obtained by removing the top-most occurrence of $e$ from stack $T$. If $e$ does not occur in $T$, then $T$ is returned.

Lastly, let $p s=\left\{c g_{1}, \ldots, c g_{n}\right\}$ be a collection of sets of propositions (e.g., possible common grounds) and let $P=\left\{p_{1}, \ldots, p_{m}\right\}$ be a set of propositions. Then define $p s \cup P=\left\{c g_{i} \cup\left\{p_{j}\right\} \mid 1 \leq i \leq n, 1 \leq j \leq\right.$ $m\}-\left\{c g^{\prime} \mid c g^{\prime}\right.$ is inconsistent $\}$. Thus the result of the operation $p s \amalg P$ is to create a new collection of sets of propositions, where each new set is formed by adding one of the elements of $P$ to an existing $c g_{i}$. At the end, any new sets that are inconsistent are discarded. Finally, we also assume an operator that rebuilds the projected set $p s$ from the current $c g$ and the contents of the Table $T$ by iteratively performing the $p s$ changes associated with each item on the Table. This operation is needed after a non-canonical conversational move is made, such as retracting an assertion, agreeing to disagree or withdrawing a question. The definition of the move is straightforward and omitted here.

\section{Default Assertions and Default Polar Questions}

In this section we characterize default assertions and default polar questions in order to be able to capture the details of their similarities and differences. We take it that any approach that cannot capture these similarities and differences cannot explain why there is overlap in the range of reactions to them and why this overlap is only partial.

\subsection{Default Assertions}

We assume, following a large body of literature, that it is useful to include assertions in the repertory of speech acts one defines. Obviously, there are many subtypes of assertions, and the complex issue of drawing their typology is not within our aims. We take it that a default assertion is performed when a participant $X$ utters a declarative sentence $S$ with falling intonation. Some assertions are non-default even if they conform to this characterization if they place specific demands on the input context. We discuss one such non-default assertion type in Section 5. Other non-default assertions, which are not addressed here, are 'rising declaratives' as well as assertions followed by interrogative tags.

We assume that declarative and interrogative sentences are distinguished by the sentential features [D] and $[\mathrm{I}]$ respectively. We treat these features as sentential markers because it is crucial to what follows for the sentence radical to which they attach to be accessible in subsequent discourse. Furthermore, we assume, in line with Hamblin (1971), that the denotation of a declarative sentence $S[D]$ is the singleton set containing the proposition denoted by its sentence radical.

Let us begin with the simple context state $K_{1}$ given in (7) in which the discourse commitment lists of

the two participants, $\mathrm{A}$ and $\mathrm{B}$, are empty, and so is the Table. The $p s$ of $K_{1}$ includes only $s_{1}$, the initial common ground at the start of the conversation. 


\begin{tabular}{|c|c|c|}
\hline A & \multicolumn{3}{|c|}{ Table } & B \\
\hline & \multicolumn{3}{|c|}{} \\
\hline Common Ground & $s_{1}$ & Projected Set $\quad p s_{1}=\left\{s_{1}\right\}$ \\
\hline
\end{tabular}

Suppose now that A asserts the sentence Sam is home, with propositional content $p$ against input context $K_{1}$. The main effects of A's assertive move are the following: (i) $p$ is added to $D C_{A}$; (ii) the syntactic structure representing this sentence and its denotation, $\{p\}$, are entered on the top of the Table stack; (iii) confirmation is projected at the level of $p s$ by adding $p$ to each element of the input $p s$. The output context state $K_{2}$ after A's move is given in (8):

$$
K_{2} \text { : A asserted Sam is home relative to } K_{1}
$$

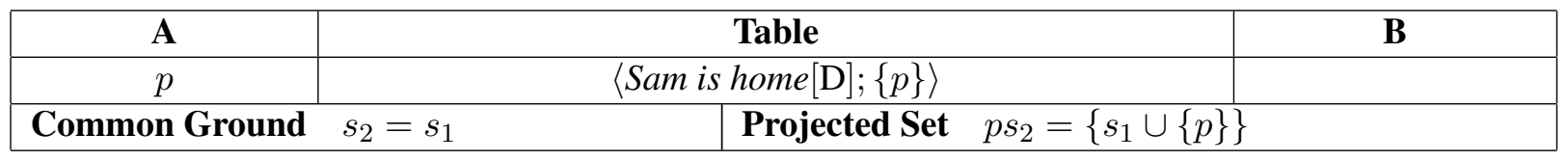

The $c g$ of the output context, $K_{2}$, is the same as that of the input context, $K_{1}$. The change involves the author's discourse commitment list, the Table, and, consequently, the $p s$.

Further conversational moves must now attend to the item on the top of the Table stack and eventually remove it from there in order to reach a stable state. The privileged future state projected by the assertion act is one whose $c g$ is updated with the asserted proposition. Projected confirmation, registered at the level of the $p s$, is the conversational goal an assertion steers the conversation toward.

We follow Krifka (2001) (see also Ginzburg (forthcoming)) in assuming that there are speech act operators that take sentences as arguments, and that are functions from input context states to output context states. We define the default assertion operator $\mathbf{A}$ as a function from an input context $K_{i}$ to an output context $K_{o}$, as in (9), where $a$ is the author of the assertion, $D C_{a, o}$ and $D C_{a, i}$ are $a$ 's output and input context discourse commitments, and $T_{o}, T_{i}$ are the output and input Table, respectively:

$$
\begin{aligned}
& \text { A }\left(S[\mathrm{D}], a, K_{i}\right)=K_{o} \text { such that } \\
& \text { (i) } D C_{a, o}=D C_{a, i} \cup\{p\} \\
& \text { (ii) } T_{o}=p u s h\left(\langle S[\mathrm{D}] ;\{p\}\rangle, T_{i}\right) \\
& \text { (iii) } p s_{o}=p s_{i} \cup\{p\}
\end{aligned}
$$

(Those elements of $K_{o}$ that are unaffected by the move have not been listed.) The change in (i) is common to all types of assertions, default or not. The other two are part of all assertion types we discuss here as well except for an assertion that simply confirms a previous assertion, in which case these two changes are fully redundant.

A default assertion then commits its author to the propositional content of the asserted sentence, it raises an issue by placing an item on the Table, and it directs the conversation towards a unique resolution of that issue, namely confirmation of the assertion. The 'issue' is the denotation of the sentence radical added to the Table in the course of the assertion. Given the effect of default assertions on their author's discourse commitment list and on the $p s$, an assertion yields an output context that is categorically biased in favor of confirmation of the asserted proposition.

The changes A brings about echo those in Poesio \& Traum (1998), where an assertion is defined as 
committing the speaker to the belief in its propositional content, while simultaneously attempting to get the addressee to believe that content as well. The main difference is that we are considering here changes triggered by particular linguistic forms that target particular conversational components, and thus, they are independent of participants' actual beliefs and intentions. The author of a default assertion may in fact aim at provoking her interlocutor to contradict her, or she may simply wish to publicize a commitment or, more radically, she might be in cahoots with her interlocutor and aim at misleading an eavesdropper or have other complex agendas. Here we are interersted in those conversational effects that are constant across all these situations and which, in our view, are connected to sentence form, prosodical properties and the input conversation state. We thus define speech acts in terms of their Heimean context change potential (CCP) rather than in terms of the actual intentions of speakers.

We follow here Stalnaker's suggestion that a side-effect of any speech act is to register in the common ground the fact that the speech act in question has occurred. Consequently, as a side effect of an assertion the common ground is automatically updated with the proposition that the author of the assertion has made the assertion. We will ignore this secondary effect of speech acts in what follows.

For Stalnaker, the essential context change associated with assertions is the addition of $p$ to the common ground. The parallel change for us is the addition of $p$ to the elements of the $p s$. Not changing the current common ground directly leaves room for moves that react to assertions, moves that have been long recognized and discussed in the literature on dialogue.

Our view that assertions raise an issue by entering an item on the Table follows earlier proposals, and is closest to Ginzburg's. For us, just as for Ginzburg, asserting a sentence with propositional content $p$ raises the issue of whether $p .{ }^{11}$ In our approach, however, it does so in a way that differs significantly from how the issue is raised when the corresponding polar question is asked, as we will see shortly. The addition of the asserted proposition to the author's discourse commitment list is the essential feature of declarative updates in Gunlogson (2001), which we import here.

Assertions project confirmation and therefore the move of confirming an assertion is the least marked next discourse move. The ability of separating confirmation from all other possible moves that follow a default assertion is a feature we exploit below in capturing differences between reactions to assertions and reactions to polar questions.

Note that what we call here default assertions may initiate a discourse but they may also connect to previous discourse by a variety of rhetorical relations of the type that form the focus of Asher \& Lascarides (2003), such as Elaboration or Counterevidence. The view of assertion given above is compatible with their participating in these rhetorical relations but the details of how that happens is not within the scope of this paper. Note that the veridicality of assertions, a property that is crucial in Asher \& Reese (2007), follows from the first and third change in (9) above.

Finally, let us note that assertions, as well as questions and other speech acts, are associated not only with literal content but with implicated content as well. We suggest that implicated content is added on the Table conjoined with but separated from the literal content making it possible for further moves to react to both implicated and literal content. One difference between the two types of content is that only literal content is associated with syntactic material on the Table, and therefore we predict differences between them when it comes to discourse ellipsis. In what follows we are concerned with literal content only.

Leaving our discourse in suspense for now with respect to whether Sam is home, we turn in the next subsection to polar questions.

\footnotetext{
${ }^{11}$ Ginzburg (forthcoming), entertains both the possibility of assertions always raising an issue and the possibility of their doing so only in case the interlocutors do not immediately confirm it.
} 


\subsection{Default Polar Questions}

In this section we consider how the context changes when a polar question speech act is performed, i.e. when a speaker utters a polar interrogative sentence such as (10):

Is Sam home?

Syntactically, we represent polar interrogatives as $S[\mathrm{I}]$ and assume that $S$ and its denotation, $p$, are available for discourse manipulation. The denotation of $S[\mathrm{I}]$ is $\{p, \neg p\} .^{12}$

When a participant A asks a polar question $S[\mathrm{I}]$, where $S$ denotes $p$, A raises the issue whether $p$ by placing on the conversational Table the interrogative sentence and its denotation, namely $\{p, \neg p\}$. Placing a question on the Table steers the conversation towards a state in which the question is resolved. For us, this means that the effect of asking a question on the input $p s$ is to add to each of its elements each of the elements in the denotation of the question.

Note that the effect of assertions and questions on the Table and on the $p s$ is identical in the sense that in both cases a sentence and its denotation are added to the Table and each element of the denotation of the sentence is added to each element of the input $p s$. The differences follow from the fact that the denotation of the declarative sentence is a singleton set while that of an interrogative is not (cf. Hamblin (1971) and Groenendijk (2003)).

Assuming that in the input context state both the Table and the participants' discourse commitment lists are empty, as in (7), the context state after asking the polar interrogative sentence Is Sam home? is as illustrated in $(11)^{13}$ :

$$
K_{4}: \text { Is Sam home? was asked relative to the initial input context } K_{1}
$$

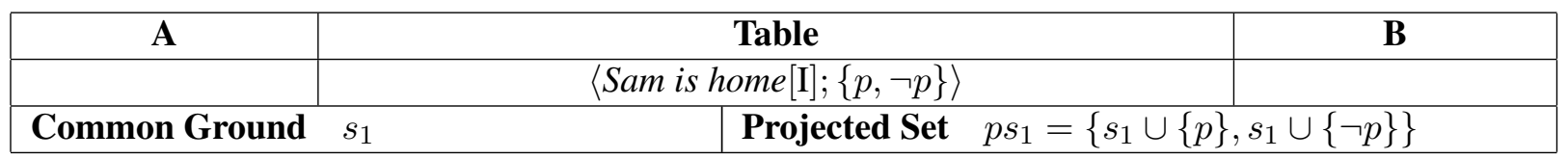

We define the polar question operator $\mathbf{P Q}$ as in (12), where $S[\mathrm{I}]$ is a polar interrogative sentence, and where $p$ is the denotation of $S$ :

Polar question operator $(\mathbf{P Q})$

$\mathbf{P Q}\left(S[\mathrm{I}], K_{i}\right)=K_{o}$ such that

(i) $T_{o}=\operatorname{push}\left(\langle S[\mathrm{I}] ;\{p, \neg p\}\rangle, T_{i}\right)$

(ii) $p s_{o}=p s_{i} \varpi\{p, \neg p\}$.

The context state after a default polar question is inquisitive with respect to the denotation of the sentence radical the move has placed on the Table because the $p s$ contains both future common grounds to which $p$ was added and future common grounds to which $\neg p$ was added. The most immediate way in which such an inquisitive context is returned to a stable state is by the addressee providing a complete direct answer to the question followed by a confirmation of this answer by the participants in the conversation, which in turn leads to the addition of the answer to the $\mathrm{cg}$. If this happens, the common ground of the conversation

\footnotetext{
${ }^{12}$ Following Karttunen (1977) we assume that the denotation of an interrogative sentence is the set of its complete answers.

${ }^{13}$ Our discourse structures do not keep track of who placed a particular item on the Table because nothing we discuss here is sensitive to this aspect. Adding such a mechanism would not cause any problems we are aware of. Similarly, adding information with respect to who acts as Source for a commitment, as argued for in Gunlogson (2008), is perfectly compatible with our proposals.
} 
becomes one of the common grounds projected by the question.

Conversational moves following a (polar) question then have to attend to getting the question removed from the Table. The goal of the conversation after the registering of the question is to resolve it by reaching a conversational state whose common ground is an element in the current $p s$.

Default polar questions are non-biasing in the sense that they do not commit their author to either proposition in their denotation and project an inquisitive context with respect to their sentence radical. There are, however, non-default polar questions that are not impartial. In English, polar interrogatives involving external negation, as exemplified in (13) have been treated as involving some sort of pre-existing bias on the part of the questioner in favor of the proposition in the scope of the negative operator, i.e., in this case, in favor of Sam being, in fact, at home.

(13) Isn't Sam home?

The issue of question bias is much debated in the literature (see, for instance, van Rooy \& Safarova (2003), Romero \& Han (2004), and references therein). We cannot add anything substantive to this debate and will not deal with external negation questions here. ${ }^{14}$

Note that the two speech acts we are concerned with in this paper contrast in both whether they affect author commitment and in how they affect the $p s$. Default assertions add to author commitment and project a non-inquisitive $p s$. Default polar questions do not add to author commitment and project an inquisitive $p s$. Non-default assertions and non-default polar questions can depart from their default counterparts in either property. In order to characterize the many types of special assertions and questions and their hybrids further finer distinctions are needed.

The most often discussed non-default questions are those involving some type of bias. Here we mention another non-default question, special in that it indicates that settling the issue is not necessarily a projected conversational future, and therefore that answering the question is optional. In Romanian, such questions are signaled by the presence of the particle oare:

$$
\begin{aligned}
& \text { Oare Petru a sosit deja? } \\
& \text { oare Peter has arrived already } \\
& \text { Has Peter arrived already? }
\end{aligned}
$$

We can accommodate oare questions in our system by assuming that the output $p s$ includes not only projected common grounds in which the question is decided but also a copy of the elements of the input $p s$ thereby indicating that not answering the question is one of the projected discourse futures. Such questions are special in that for them removal from the Table without change in the current $c g$ is a projected option. Our characterization correctly predicts that oare questions are not appropriate on forms, as exam questions or as questions asked in court or by a policeman.

Before concluding, we mention a welcome consequence of our approach to polar questions. Having the uttered sentence entered on the Table allows us to differentiate between positive and negative polar questions while at the same time assigning them the same denotation. An (inner negation) negative polar question such as:

\section{(15) Is Sam not home?}

\footnotetext{
${ }^{14} \mathrm{We}$ assume that questions that introduce strong bias are non-default. We come back to weak bias involved in positive and in inner negation polar questions in Section 4.2.
} 
has the same effect on the ps as its positive counterpart. It differs from it, however, in that it places a negative rather than a positive sentence on the Table. Pragmatic differences between positive and negative polar questions as well as differences in the way they can be answered are connected, in our view, to the difference in the contribution the two types of questions make to the Table without having to give up the uniform account of the denotation of affirmative and negative questions.

To sum up, the major differences between default assertions and default (polar) questions under the view presented above are: (i) default assertions upgrade their author's commitment list while default (polar) questions do not; (ii) default assertions enter a declarative sentence on the Table, while polar questions enter an interrogative sentence; (iii) at the level of the $p s$, default assertions result in a non-inquisitive context whereas default questions result in an inquisitive one. The major similarity between asserting a sentence $S[\mathrm{D}]$ and asking the corresponding polar question by uttering $S[\mathrm{I}]$ is that the same sentence radical, $S$, is placed on the Table. The major difference between $S[\mathrm{D}]$ and $S[\mathrm{I}]$ is that the denotation of a former is a singleton and that of the latter is not. In both cases the sentence radical is decided relative to each element of the output $p s$. Reactions to these two speech acts, we predict, will be sensitive to this common denominator as well as to the differences involved. These predictions are explored in the rest of the paper.

\section{Reacting to default assertions and default polar questions}

A default assertion and a corresponding polar question (our Sam is home and Is Sam home? examples) raise the same issue: that of deciding the status of the proposition expressed by the sentence radical they place on the Table. In this section we characterize two main conversational move types that deal head on with the issue raised, confirmation and reversal. This sets the stage for the discussion of responding assertions and polarity particles in the next section.

\subsection{Confirming and reversing an assertion}

Let us turn back to the aftermath of an assertion, and assume that the current context state is as in (8). The least marked reaction to an assertion is confirmation, a move that commits its author to the propositional content of the sentence on the Table. We define in (16) an explicit assertion confirmation operator, AC, as a function from an input context $K_{i}$ to an output context $K_{o} . S[\mathrm{D}]$ is a declarative sentence with denotation $\{p\}$ that has been asserted by some discourse participant $a$ in a previous move and is currently at the top of the Table, as well as on $a$ 's commitment list in $K_{i}$. Let $b$ be the participant confirming the assertion and let $D C_{b, o}$ and $D C_{b, i}$ stand for $b$ 's discourse commitments in the output and input context states respectively.

\section{Assertion Confirmation (AC)}

a. Input context conditions:

(i) $\operatorname{top}\left(T_{i}\right)=\langle S[\mathrm{D}] ;\{p\}\rangle$

(ii) $p$ in $D C_{a, i}$

b. Change:

$\mathbf{A C}\left(b, K_{i}\right)=K_{o}$ where $D C_{b, o}=D C_{b, i} \cup\{p\}$

The fact that $p$ is an element of some discourse commitment list follows from $S$ [D] being on the Table. If the author of the confirmation is the same participant as the author of the assertion he is confirming, the confirmation move is informationally redundant. Such a redundant move may be used for adding emphasis or for other rhetorical purposes. 
After all participants in a conversation confirm an assertion, $p$, the proposition denoted by the asserted sentence radical, is present on the commitment list of each participant. We assume that in this case an auxiliary move $M^{\prime}$ applies, whose effects are given in (17).

\section{Common ground increasing operation $M^{\prime}$}

If an operator $M$ contains a change of the form $D C_{X, o}=D C_{X, i} \cup\{p\}$, and, as a result, $p$ is now present on the commitment list of each participant in the conversation in $K_{o}$, add the following changes to $M$ :

1. $c g_{o^{\prime}}=c g_{i} \cup\{p\}$,

2. $D C_{X, o^{\prime}}=D C_{X, o}-\{p\}$ for all participants $X$.

3. Pop off of the top of the Table all items that have as an element of their denotation an item $q$ that is entailed by $c g_{o^{\prime}}$.

$M^{\prime}$ adds the proposition that is present on each commitment list to the $c g$ and removes it from individual commitment lists. We assume that if any of the propositions in the denotation of an item on the Table are decided relative to the $c g$ of the conversation, that item is popped off from the Table, and therefore items from the Table that are decided as a result of the addition of $p$ to the $c g$ are popped off the Table as part of $M^{\prime}$.

Confirmation is the default reaction to an assertion because the change it performs is a necessary step towards reaching the $c g$ projected by the assertion and thus canonically settling the issue raised. Assertion confirmation can be signaled in a variety of linguistic and non-linguistic ways. One can nod, say $u h$ huh, sure, right, you bet, yup, etc. One can also express assertion confirmation by reasserting the asserted proposition, as in (18), using a more or less truncated form.

Anne: Sam's home.

Ben: Yes, he is.

We call the type of assertion exemplified in Ben's utterance above an responding assertion and we discuss it in detail in the next section. There are many other ways of indicating assertion confirmation, some of which involve implicature. (For discussion, see Walker (1996).) Note that assertion confirmation may be left unsignaled altogether. This is, we claim, unsurprising given the default nature of the move, under the natural assumption that the more canonical a move is the less overt marking it needs. ${ }^{15}$ In what follows we only deal with overt assertion confirmation moves.

Finally, note that what was said so far distinguishes between the three assertions in the short conversation in (19), even though they are identical with respect to their propositional content:

Anne: Sam is home.

Ben: Yes, he is.

Anne: (Yes,) Sam is home.

Assuming that Anne's first assertion occurs in a context in which Sam's whereabouts were not yet discussed, it raises the issue of whether Sam is home or not, and commits Anne to the claim that he is. Ben's assertion signals confirmation, a non-redundant move; the proposition expressed by Sam is home is now on Ben's commitment list as well. If this is a dialogue between Anne and Ben, the issue is resolved and $M^{\prime}$ applies.

\footnotetext{
${ }^{15}$ While silence can be taken as assertion confirmation, we do not suggest that its overall impact is identical to that of a responding assertion or some other overt confirmation marker. Confirmation by failure to object is the weakest possible way of signaling conversational commitment. Silence can be seen as implicating confirmation based on Walker's Collaborative Principle.
} 
Anne's second assertion, on the other hand, is redundant. It recommits Anne to a proposition she just committed herself to and, if not a reactive move, it raises an issue that has just been settled.

Assertion confirmation decides the issue raised by an assertion as far as the author of the confirmation is concerned, in the direction of the proposal made by the original assertive move. Another way of deciding the issue for the author of the reactive move is to reverse the asserted proposition in toto or partially. We call assertion reversing moves total or partial denial. A total denial is exemplified by Ben's first reaction; his second illustrates partial denial:

Anne: Mary ordered chicken yesterday.

Ben: No, she didn't./No, it was beef.

We follow van der Sandt \& Maier (2003) in taking denials to be special conversational moves that commit their author to the complement of the denotation of a declarative sentence placed on the Table by the preceding move. Denials are highly marked and therefore they must be explicit and must be signaled, Walker (1996) argues, as early as possible. As already mentioned in Section 1, denials are marked moves in our terms because the proposition the author of a denial commits to cannot become a joint commitment in a coherent discourse without retraction on the part of another participant, and therefore the issue placed on the Table by the initial assertion cannot be removed in a canonical way.

We restrict our attention here to denials expressed by an assertion, as in the examples above. Ben's two moves are assertions, i.e., they commit their author to the proposition denoted by the asserted sentence, call it $p^{\prime}$ and they place that sentence and its denotation on the Table. The special nature of these assertive moves resides in the fact that they react to a previous assertion whose propositional content is inconsistent with $p^{\prime}$. The $p s$ of the conversational state that results after they have been made is empty, and the conversation is in crisis. Total denials contradict the previously asserted proposition; partial denials contradict a subpart of what has just been asserted, but commit their author to everything else that the previous assertion contributed. As noted by van der Sandt and Maier, partial denials must have access to the totality of the message conveyed by the assertion they react to, its entailments, presuppositions, and conversational as well as conventional implicatures. ${ }^{16}$

We narrow our focus further to total denials. The starkest form of total denial is to assert the opposite of the previously asserted sentence. Such a situation is given in (21), where $s_{2}$ is the common ground of the discourse state $K_{2}$ from (8) that served as the output from A's assertion:

$$
K_{4} \text { : Denial on the Table }
$$

\begin{tabular}{|c|c|c|}
\hline $\mathbf{A}$ & Table & B \\
\hline$p$ & $\langle S[\mathrm{D}] ;\{p\}\rangle$ & $\neg p$ \\
& $\langle\neg S[\mathrm{D}] ;\{\neg p\}\rangle$ & \\
\hline Common Ground & $s_{4}=s_{2}$ & Projected Set $\quad p s_{4}=\emptyset$ \\
\hline
\end{tabular}

Denial moves may be performed by assertions, as was the case in our example, or by various reversing markers such as no way, definitely not, etc. Denying responding assertions, exemplified by Ben's assertion above will be examined in detail in the next subsection.

We define below a total denial assertive move as one that reacts to a previous move whereby $p$ was asserted, and which asserts a sentence $S^{\prime}$ whose propositional content is $\neg p$.

\footnotetext{
${ }^{16}$ See also Asher \& Lascarides (2003) for insightful discussion.
} 
a. Input context conditions:

(i) $\operatorname{top}\left(T_{i}\right)=\langle S[\mathrm{D}] ;\{p\}\rangle$

(ii) $p$ in $D C_{a, i}$

b. Change:

(i) $\mathbf{T D}\left(b, K_{i}\right)=K_{o}$ where $D C_{b}=D C_{a} \cup\{\neg p\}$

(ii) $T_{o}=\operatorname{push}\left(\left\langle S^{\prime}[\mathrm{D}] ;\{\neg p\}\right\rangle, T_{i}\right)$

When denial does not involve an overt assertion, the changes are the addition of $\neg p$ to the author's discourse commitment list and to the Table.

In the aftermath of a denial, one can eventually reach a stable state in one of two ways: (i) one of the participants retracts their assertion; or (ii) the participants 'agree to disagree'. We deal here only with the latter because it underscores the need to separate participant commitments from the $c g$, a point we insisted on in Section 2. Besides, the complexities raised by retractions go beyond the scope of this paper.

Let us assume that A asserted $S[\mathrm{D}]$, where $S$ denotes $p$, and B responded by asserting $S^{\prime}[\mathrm{D}]$, where $S^{\prime}$ denotes $\neg p$. The move of 'agreeing to disagree' involves removing $\langle S[\mathrm{D}] ;\{p\}\rangle$ and $\left\langle S^{\prime}[\mathrm{D}] ;\{\neg p\}\right\rangle$ from the Table without removing either $p$ or $\neg p$ from the relevant commitment lists. Each participant remains publicly committed to the propositional content of whatever they asserted, but neither $p$ nor $\neg p$ is added to the $c g$. The state of the conversation after the move of agreeing to disagree has been carried out in our example is as in (23):

$$
K_{5}: \mathrm{A} \text { and } \mathrm{B} \text { have agreed to disagree relative to } K_{4}
$$

\begin{tabular}{|c|c|c|}
\hline $\mathbf{A}$ & \multicolumn{3}{|c|}{ Table } & B \\
\hline$p$ & \multicolumn{3}{|c|}{} & $\neg p$ \\
\hline Common Ground $\quad s_{5}=s_{4}$ & Projected Set $\quad p s_{5}=\left\{s_{5}\right\}$ & \\
\hline
\end{tabular}

The common ground in $K_{5}$ is identical to the common ground in $K_{4}, K_{3}$, and even $K_{1}$, the context state that served as input to the move in which A asserted $S[\mathrm{D}]$. Under the assumption that that common ground, $s_{4}$, was consistent and that it was consistent with both $p$ and $\neg p$, the context state $K_{5}$ is consistent at every level and therefore the conversation is no longer in crisis, even though the commitment lists of the two participants in $K_{5}$ are mutually inconsistent. Separating commitment lists from the $\mathrm{cg}$ is crucial in capturing the fact that, after a move of agreeing to disagree, the conversation is not in crisis. In future context states, however, in order for A to remain coherent, her public commitments have to be consistent with $p$, while in order for B stay coherent, his public commitments have to be consistent with $\neg p$. The move of agreeing to disagree requires an input context that has a denial on the top of the Table. The operation it performs is to remove the contradictory items from the Table and reconstruct the $p s$ based on what remains on the Table. Such a move is usually proposed by one of the participants by uttering an imperative such as Let's agree to disagree/Let's not pursue this further. It is carried out after the other participant(s) agree to comply with the imperative. The imperative in this case is special in that compliance with it involves a change in the context state of the conversation.

\subsection{Confirming and reversing a polar question}

Recall that default polar questions, just like assertions, place a sentential issue on the Table. Unlike assertions, however, they do not commit their author to a particular resolution of that issue and they project 
acceptance of both the denotation of the sentence radical and of its complement.

After a polar question has been placed on the Table, the canonical way of removing it is by deciding the issue raised. In our example in (11), this means deciding whether Sam is home or not. The most direct way of achieving this is by adding either $p$ or $\neg p$ to the current $c g$, where $p$ is the denotation of the sentence radical the polar question has placed on the Table. We address separately these two possible responses, as the distinctions will be useful to us later.

We call polar question confirmation the move that reacts to a polar question by committing the author to the propositional content of the sentence radical on the Table. We define it in (24).

\section{P-Question Confirmation (P-QC)}

a. Input context condition: $\operatorname{top}\left(T_{i}\right)=\langle S[\mathrm{I}] ;\{p, \neg p\}\rangle$

b. Change:

(i) $\mathbf{P}-\mathbf{Q C}\left(b, K_{i}\right)=K_{o}$ where $D C_{b, o}=D C_{b, i} \cup\{p\}$, where $p$ is the denotation of $S$.

(ii) $T_{o}=\operatorname{push}\left(\langle S[\mathrm{D}] ;\{p\}\rangle, T_{i}\right)$

Polar question confirmation is similar to assertion confirmation in that the author of such a move commits to the propositional content of the sentence radical on the Table. Once the other participants in the conversation also commit to this proposition, the issue is decided and $M^{\prime}$ applies, adding $p$ to the $c g$, and removing the relevant items from the Table and from the commitment lists of the participants. Therefore, it comes as no surprise that the two types of acceptance moves share responses. In English, polar-question confirmation, just as assertion confirmation, can be signaled by nodding, by saying $u h$ huh as well as by assertions that repeat the previous assertion, as exemplified in (25):
A: Is Sam home?
$\mathrm{B}$ : Yes, he is.

When a polar question confirmation move is made by an assertion, the asserted sentence as well as its propositional content are added to the Table. Note that the addition is not redundant in this case because it affects the input $p s$ leading to the elimination of those elements that are inconsistent with the denotation of the sentence radical.

Generalizing over assertion confirmation and polar question confirmation, we characterize a type of discourse move we call confirming in (26):

\section{Confirming move type}

a. Input context condition contains:

top $\left(T_{i}\right)=$ item whose sentence radical denotes a proposition $p$

b. Change contains:

$$
D C_{a, o}=D C_{a, i} \cup\{p\}
$$

A move is confirming iff it reacts to an immediately previous move that has placed a proposition denoting sentence radical on the Table, and the move commits its author to that proposition. Both assertion confirmation and polar question confirmation are confirming moves. Assertions that qualify as confirming move types will be called confirming assertions.

Confirming a polar question is crucially different from confirming an assertion because in the case of polar questions, confirmation is not the only projected outcome in the input context: reversing is also projected. This, we claim, is responsible for the fact that assertion confirmation can be left completely 
implicit, while polar question confirmation can not. Silence after a default polar question cannot be normally taken as confirmation.

Note that a polar question confirmation move does not necessarily lead to agreement on the issue at hand between the author of the question and the author of the confirmation move since the former has not publicly committed to either outcome. Such agreement arises only after the author of the polar question confirms the resolution proposed by the author of the confirmation move. Assuming that Anne and Ben are the only participants in the conversation in (25) above, the issue of whether Sam is home will be decided in favor of him being in fact home only after Anne confirms Ben's assertion. In the most common case, that of information seeking questions, the questioner is assumed not to know the answer to her question and to assume that the addressee does, and therefore her confirmation of the addressee's answer is even less marked than in the case of ordinary assertions.

Reversing a polar question, just like in the case of reversing an assertion, involves committing to $\neg p$, the complement of the proposition expressed by the sentence radical on the top of the input Table. In the case of polar questions, such a move is not a denial, however, and it does not result in a crisis because a default polar question does not categorically bias the context in favor of one resolution versus another, the way assertion does. Ben's reactions in (27), therefore, does not result in crisis:

Anne: Is Sam home?

Ben: No, he isn't.

The move of polar question reversing is defined below:

P-Question Reversing (P-QR)

a. Input context condition:

$\operatorname{top}\left(T_{i}\right)=\langle S[\mathrm{I}] ;\{p, \neg p\}\rangle$

b. Change:

(i) $\mathbf{P}-\mathbf{Q R}\left(b, K_{i}\right)=K_{o}$ where $D C_{b, o}=D C_{b, i} \cup\{\neg p\}$, where $p$ is the denotation of $S$.

(ii) $T_{o}=\operatorname{push}\left(\langle\neg S[\mathrm{D}] ;\{\neg p\}\rangle, T_{i}\right)$

Generalizing over what is common in moves that reverse assertions and polar questions we define a move type we call reversing as in (29):

Reversing move type

a. Input context condition contains:

$\operatorname{top}\left(T_{i}\right)=$ item whose sentence radical denotes a proposition $p$

b. Change contains:

$D C_{a, o}=D C_{a, i} \cup\{\neg p\}$

Reversing moves are reactions to immediately previous moves that place a proposition denoting sentence radical on the Table, and commit their author to the complement of that proposition. Common to denials and polar question reversal is that they are reversing moves. The difference between them is due to the differences in the input context: in the case of denials, the input context is categorically biased in favor of the proposition denoted by the sentence radical on the input Table top, while in the case of polar question reversal, this is not so. Assertions that have the property of being reversing moves will be called reversing assertions. ${ }^{17}$

\footnotetext{
${ }^{17}$ We have defined here only total reversal. For partial reversal we require the author of the move to commit to a proposition that, together with the current $c g$, is inconsistent with the denotation of the sentence radical on the top of the input Table (or one of its
} 
Confirming moves are formally simpler than reversing moves because the relation they involve is identity rather than complementation. If we take this into account, the answers polar questions project are not identical even in non-biased questions, since confirming answers are less marked than reversing ones. This allows us further distinguish positive and negative polar questions. If we assume that polar questions involve a weak bias towards the least marked answer we capture the weak bias of positive polar questions towards a positive answer, and the weak bias of negative polar questions towards the negative answer noticed in Rooy \& Safarova (2003).

Just as agreeing to disagree is a non-canonical way of removing an assertion from the Table, one can agree not to pursue a question if there is no agreement on an answer, thereby removing it from the Table without having resolved it. Such a move is non-canonical because the question is popped off the stack without any concomitant enrichment of the common ground. Once the question is removed, the projected set must be recalculated. Such moves may be prompted by participants declaring their inability to provide information that would lead to answering the question.

Generalizing now over confirming and reversing moves we define in (30) a move type we call responding:

\section{Responding move type}

a. Input context condition contains:

top $\left(T_{i}\right)=$ item whose sentence radical denotes a proposition $p$

b. Change contains:

$$
D C_{a, o}=D C_{a, i} \cup\{p\} \text { or } D C_{a, o}=D C_{a, i} \cup\{\neg p\}
$$

Responding moves commit their author to the denotation of a sentence radical on the Table, or to its complement.

\subsection{Responses to assertions vs. responses to polar questions}

We conclude this section by looking back at the similarities and differences between responses to assertions and responses to polar questions.

We call here responding assertions those assertions that perform a responding move, and initiating assertions those subtypes of assertions that are not responding. Since initiating assertions and polar questions place an issue on the Table in the form of a proposition-denoting sentence radical, moves that react to them are can be responding and therefore confirming or reversing. Since constituent questions do not place a proposition-denoting sentence radical on the Table, reactions to such questions can be neither confirming nor reversing.

In order to capture the common denominator of respoinding moves we propose to introduce two relative polarity features, [same] and [reverse], the former marking confirming moves and the latter marking reversing ones. They are defined in (31):

(31) a. A responding move has the feature [same] if and only if it is a confirming move.

b. A responding move has the feature [reverse] if and only if it is a reversing move.

Recall that the crucial difference between assertions and (polar) questions is that the former, but not the latter, commit their author to the propositional content of the asserted sentence. It therefore follows that confirmation in the case of assertions but not in the case of polar questions amounts to agreement. By

implicatures). Partial reversal can be made directly or by implicature. 
the same token, reversing in the case of assertions, but not in the case of polar questions, amounts to denial. Given these observations, we predict partial overlap when it comes to the form of responding moves reacting to assertions and polar questions. We expect overlap as far as the responding nature of the move is concerned. The expected differences should be ultimately connected to the differences in the state of the input context to the reactive move. We discuss these predictions in more detail in the next section, where we focus on responding assertions.

\section{Responding assertions and polarity particles}

In this section we narrow our attention to responding assertions of the type illustrated in Ben's assertions below:

Anne: Sam's home.

Ben: Yes, he is./No, he isn't.

Anne: Is Sam home?

Ben: Yes, he is./No, he isn't.

These are the most direct responding assertions possible in that they assert the denotation of the sentence radical on the top of the input Table or its opposite.

\subsection{Responding assertion typology}

The category of responding assertions extends the notion of answers to questions discussed briefly in Sadock \& Zwicky (1985), pp. 189 - 191, so as to cover reactions to assertions as well as reactions to questions. As such, it cannot be seen as a subtype of the Question-Answer Pair (QAP) rhetorical relation discussed in Asher and Lascarides' work. That notion is not general enough to capture the fact that responding assertions span reactions to polar questions and assertions. On the other hand, QAP is meant to cover answers to all types of questions, not only polar ones, and therefore it is too wide for our purposes. The formal parallelism between responding assertions responding to assertions and those responding to questions, on the other hand, points to the need for the category we provide.

Responding assertions can be differentiated based on three criteria: (a) whether they respond to an assertion or a polar question; (b) whether they are confirming or reversing; (c) whether the sentence they assert is positive or negative.

The first criterion concerns the status of the input context. In the case of responses to assertions, the input context is already categorically biased in favor of the proposition denoted by the sentence radical. At least one of the participants is committed to that proposition and all elements of the input $p s$ already have that proposition as an element. ${ }^{18}$ In the case of responses to polar questions, no such categorical bias has been brought about by the item on top of the Table in the input context. Normally, the input $p s$ contains both elements that decide the issue in favor of the denotation of the sentence radical and elements that decide the issue in favor of the complement of that proposition. ${ }^{19}$

The second criterion concerns the relationship between the proposition the assertion commits its author to and the propositional content of the sentence radical on the top of the input Table. There are two possi-

\footnotetext{
${ }^{18}$ If the context is already in crisis, there are no elements in the input $p s$.

${ }^{19}$ An exception is when the answer to the question already follows from the contents of the input $\mathrm{cg}$, as is the case with rhetorical questions such as "Is the Pope Catholic?" Other types of rhetorical questions are special only in that the author of the response is assumed to be the same participant as the author of the question, as in the case of rhetorical questions asked during lectures.
} 
bilities: (i) the response is confirming, in which case it bears the relative polarity feature [same] or (ii) the response is reversing, in which case it bears the relative polarity feature [reverse].

Combining the two criteria, we get [same] and [reverse] responses to assertions and [same] and [reverse] responses to polar questions. As discussed above, [same] responses to assertions are predicted to be the least marked possible reactions. A [reverse] response to an assertion (a denial) is the most marked reaction. In the case of responses to polar questions, neither [same] nor [reverse] responses necessarily lead to crisis. We take it, however, that [same] assertions are simpler than [reverse] ones because the former assert the sentence radical already present on the top of the input Table. Thus, we expect that [same] assertions will be less marked than [reverse] assertions even when reacting to a polar question.

The third criterion is specific to responding assertions. In [same] assertions the polarity of the asserted sentence matches the polarity of the sentence radical on the Table top of the input context; in [reverse] assertions, the polarity of the asserted sentence is the reverse of that of the sentence radical on the Table top of the input context. We assume that a responding assertion that asserts a positive sentence has the absolute polarity feature $[+]$, while a responding assertion that asserts a negative sentence has the absolute polarity feature [-]. We also assume, following Horn (2001) and much literature besides, that $[+]$ assertions are less marked than $[-]$ assertions.

We illustrate our two polarity features in (34) and (35):

Anne: Sam is home./Is Sam home?

Ben: Yes he is. $([$ same,+$])$

Connie: No, he isn't. ([reverse, -$]$ )

Anne: Sam is not home./Is Sam not home?

Ben: Yes, he is. ([reverse, +$]$ )

Connie: No, he isn't. ([same, -$]$ )

In what follows we examine the role of these two polarity features in accounting for the particles that occur at the leftmost edge of sentences functioning as responding assertions.

\subsection{Polarity particles}

In this section we take a closer look at particle usage in responding assertions, and examine the parameters they are sensitive to, basing our discussion on the typology and features given in the preceding section. Our aim is to provide a glimpse of the use of these features in accounting for the distribution of polarity particles concentrating on a three polarity particle language, Romanian. The discussion illustrates how the approach we developed so far is able to capture the partial overlap between responding assertions that react to assertions and to polar questions respectively.

So far we have established that in dialogues such as (36),

(36) Anne: Sam is home.

Ben: Yes, he is./No, he isn't.

Anne: Is Sam home?

Ben: Yes, he is./No, he isn't.

Ben's utterances are responding assertions. The first answer is a [same] response, and the second is a [reverse] one. In the case in which these assertions respond to a previous assertion, the first amounts to confirmation, the second to total denial. 
In English, the particles yes and no, placed at the left edge of a declarative sentence signal the responding nature of the assertion. We treat them both as presupposing that the assertion is responding, i.e., confirming or reversing. These particles are therefore predicted to be inappropriate in assertions made relative to an input context state whose Table does not contain, on the top of its stack, a proposition the reactive assertion can be seen as confirming or reversing. We thus correctly predict that yes and no are inappropriate in initiating assertions as well as in answers to a wh-question.

The choice between yes and no in responding assertions is influenced, we claim, by the two polarity features introduced in the previous subsection. In (36) and (37), yes can be seen as marking both [same] and $[+]$, and no can be seen as marking both [reverse] and [-]. In (35), yes marks [+] and no marks [-]. These two particles can also be used to encode relative polarity only, with yes signaling [same] and no signaling [reverse]:

Anne: Sam is not home.

Ben: Yes. He is not. / No. He is.

Both intonation and context help separate these two functions of the English polarity particles. ${ }^{20}$

The particle overlap between $[+]$ and [same] on the one hand, and $[-]$ and [reverse] on the other, seen in English, is not surprising given the unmarked nature of the features [same] and $[+]$ and the marked nature of the features [reverse] and [-]. See Pope (1976) for further discussion and examples.

Recall now that a [reverse] assertion in reaction to a polar question, though intrinsically more marked than a [same] assertion, does not lead to conversational crisis. Such a move in reaction to an assertion, on the other hand, does lead to crisis. We call [reverse] assertions that react to an assertion denials and [reverse] assertions that react to a polar question, reverse answers. Denials and reverse answers share the [reverse] feature but are distinguished with respect to both their input and their output context states. Denials are conversationally marked moves because they lead to conversational crisis. Recall also that confirming reactions to assertions and confirming reactions to polar questions share the feature [same] but differ in that the former are the least marked conversational moves discussed here.

Given this partial similarity between reactions to assertions and polar questions we expect partial overlap in their form, with denials being formally more marked than reverse answers and confirming reactions to assertions being less marked than confirming reactions to polar questions. Thus, the similarities in form between Ben's moves in (36) and (37) above are due to the fact that the yes and no responses have identical relative and absolute polarity features independently of whether they react to an assertion or a polar question. The differences in the conversational states that results after Ben made his response in the two dialogues above stem from the differences in the input context state in the two cases.

The importance of the two types of polarity features of reactive assertions introduced here becomes evident once we consider the inventory of polarity particles across languages. We cannot go here into a detailed study of polarity particles within a language or cross-linguistically. We will, however, illustrate with polarity particles in Romanian, and end with some general predictions our approach makes. For details on the Romanian particle system, see Farkas (to appear).

We assume here that polarity particles, such as the use of yes and no illustrated above, are items that occur at the leftmost edge of a root sentence, in a node we christen PolP, that houses the absolute and relative polarity features of an responding assertion. The sister node of PolP is a CP whose polarity agrees, by definition, with the absolute polarity feature in PolP.

As discussed in Pope (1976), there are languages such as Japanese, that use particles to signal only

\footnotetext{
${ }^{20}$ Note also that the use of yes is correlated not only with [same] and $[+]$ but also with the author being the source of the information the move commits her to, as discussed in Gunlogson (2008).
} 
relative polarity, i.e., [same] and [reverse], while other languages use polarity particles meant to primarily signal the absolute polarity of the asserted sentence in responding assertions. ${ }^{21}$ There are also languages that have a mixed system, with some particles primarily associated with absolute polarity and others primarily associated with relative polarity. As mentioned in Section 1, Romanian is such a languate. It has three polarity particles, the positive particle $d a$, the negative particle $n u$, and a third particle, $b a$, whose function is, we claim, to signal [reverse].

We call $d a$ and $n u$ absolute polarity particles because their use is primarily sensitive to the polarity of the sentence they assert. We assume here that sentence radicals are either positive or negative, a fact encoded in their form. Recall that a responding assertion has the polarity feature $[+]$ iff its sister $\mathrm{CP}$ is positive and the polarity feature [-] iff its sister CP is negative. As seen below, the positive polarity particle $d a$ in Romanian occurs in $[+]$ responding assertions while $n u$ occurs in $[-]$ responding assertions:
A: Ana a plecat. 'Ana left.'
B: Da. / Da, a plecat. 'Yes./Yes, she left.'

A: Ana nu a plecat. 'Ana didn't leave.'

B: Nu, n-a plecat. 'No, she hasn't.'

A: Ana a plecat? 'Did Ana leave?'

B: Da. / Da, a plecat. / Nu. / Nu, n-a plecat. 'Yes./Yes, she left. No./No, she hasn't left.'

In (40) we see $n u$ in a [same, -] reaction; in (44) below we will see da in a [reverse, +] reaction.

As exemplified above, absolute polarity particles may occur on their own in Romanian, as well as in English. Responses whose only overt item is a polarity particle can be treated as involving sentential ellipsis or some sort of pro-sentential form. We treat such utterances as responding assertions but stay neutral with respect to whether ellipsis or a sentence pronominalization is involved. For an ellipsis account, see Kramer \& Rawlins (to appear), who follow Merchant (2004)).

Turning now to the third particle, $b a$, we illustrate its distribution below ${ }^{22}$ :
A: Ana a plecat. 'Ana left.'
B: Ba nu, n-a plecat./*Nu, n-a plecat. 'No, she didn't.'
A: Ana a plecat? 'Has Ana left?"
B: Nu. / Nu, n-a plecat. /*Ba nu. /*Ba nu, n-a plecat. 'No, she hasn't.'
A: Ana nu a plecat. 'Ana didn't leave.'
B: Ba da. / Ba a plecat. /Ba da, a plecat. 'You are wrong, she did.'

\section{A: Ana nu a plecat? 'Didn't Ana leave?' \\ B: Ba da. / Ba a plecat. / Ba da, a plecat.' 'Yes, she did.'}

We now turn to some generalizations drawn on the basis of the distribution of these particles. First, note that the above examples show that $b a$ functions as a signal of the feature [reverse]. It may occur only in moves marked with this feature, both as reactions to assertions and as reactions to polar questions. What follows $b a$ expresses overtly the absolute polarity of the asserted sentence, under the assumption that this can be done both by absolute polarity particles and the polarity of the verb of the asserted sentence. Under the assumption that the absolute polarity of the asserted sentence must be overtly expressed in Romanian

\footnotetext{
${ }^{21}$ In the terminology of Sadock \& Zwicky (1985) the latter languages have a yes/no system while the former have an agree/disagree system.

${ }^{22}$ Romanian, unlike English, does not have a syntactic contrast between internal and external negative questions.
} 
we account for the fact that $b a$ cannot occur on its own. ${ }^{23}$

As illustrated in (42), a responding assertion marked with $b a$ can be used to return a negative assertion when the input context presented a positive sentence, in which case $b a$ is followed by $n u$ and/or the negative asserted sentence. It can also be used (as in (44) and (45)) to return a positive sentence when the input context presented a negative one, in which case the absolute polarity is expressed by $d a$ and/or a positive entence.

So far then, we have seen that the existence and distribution of the particle $b a$ confirms the necessity of recognizing the class of [reverse] responses. The Romanian data discussed so far also points to the necessity of separating absolute and relative polarity. In characterizing relative polarity, it is crucial to be able to define confirming and reversing move types, which in turn relies on being able to make reference to the polarity of the proposition denoting sentence radical on the top of the input Table.

Recall now that our characterization of responding assertions makes a distinction between denials and reverse answers. We therefore predict the possibility of a differentiation in polarity particle distribution that is sensitive to this distinction. Furthermore, given that denials are the most marked conversational moves, we expect the reverse particle to be used in denials more readily than in reverse answers. The distribution of $b a$ in Romanian confirms these expectations. The contrast between (42) and (43) above shows that the distribution of $b a$ is sensitive to whether the move is a reaction to an assertion or a question: in assertions whose features are [reverse] and [-], $b a$ is required in denials but is not possible in reverse answers. We thus see a difference between denials and reverse answers with denials being more marked than reverse answers, as expected.

The data in (44) and (45) above show that the contrast between denials and reverse answers just noted disappears in case the absolute polarity feature of the reverse assertion is $[+]$, i.e., when the responding move reverses a negative sentence radical on the top of the input Table. It appears therefore that, at least in Romanian, the overt marking of the feature [reverse] is sensitive not only to whether the move is a denial or not but also to its absolute polarity feature. The data suggest that the combination $[$ reverse $]$ and $[+]$ calls for the use of a reverse marker more readily than the [reverse] and [-] combination.

In looking for a justification for this fact we recall the observation in Pope (1976), who notes that absolute $[+]$ particles may double to express [same] while [-] particles may double to express [reverse], as exemplified in English with yes and no. Given this affinity between [reverse] and [-] on the one hand, and [same] and [+] on the other, the combination of [reverse] and [+] appears special because its component features are not compatible. Using a single relative particle to express both features is problematic because of the impossibility of [reverse] particles to mark [+]; having recourse to a single absolute particle on the other hand is problematic because of the impossibility of $[+]$ particles to mark [reverse]. Note also that in these responses [+] is contrastive and therefore we expect it to require special marking, unlike in $[$ same, +$]$ cases. In support of the special nature of the combination of [reverse] and $[+]$ we note that in French and German the particles si and doch appear to be used in responding assertions precisely to mark the combination of these two features. Thus, the French and German 'third' particles do the job of the Romanian ba da sequence:

(46) A: Anne n'est pas partie. 'Anne didn't leave.'

B: Mais si. 'You are wrong, she did.'

A: Anne n'est pas partie? 'Didn’t Anne leave?'

B: Mais si. 'Yes, she did.'

\footnotetext{
${ }^{23}$ There are non-standard dialects where this is not so and where $b a$ may occur on its own, in which case it is interpreted as an absolute negative polarity particle.
} 
A: Anna kommt nicht mit ins Kino. 'Anna isn't coming along to the movies.'

B: Doch! Sie kommt schon. 'You are wrong. She's coming.'

A: Wollen Sie den Job nicht? 'Don't you want this job?'

B: Doch! Ich brauche das Geld. 'But I do. I need the money.'

A parallel tension exists in responding assertions whose features are [same] and [-]. In their case, a negative particle may express the absolute but not the relative feature, while a positive particle could express the relative but not the absolute feature. This combination is less problematic, however, because the absolute feature is not contrastive and the relative feature is the unmarked one. In Romanian in such cases the relative polarity remains unexpressed, a fact that is in keeping with [same] being the unmarked relative polarity feature. In English the combination yeah no can be used for just such cases:

Anne: Sam is not home. / Is Sam not home?

Ben: Correct./Yup./Yeah no. ${ }^{24}$

Finally, note that the fact that in Romanian the third particle is used to mark [reverse] rather than [same] is unsurprising given the fact that reversing moves are more marked than confirming ones.

The brief glimpse of polarity particles this subsection has provided has shown, we hope, the usefulness of the two types of polarity features we have identified as characterizing responding assertions. In defining relative polarity features we have relied on the material in Sections 3 and 4, which allows us to capture the similarities and differences between reactions to assertions and polar questions and in characterizing responding move types. These sections, in turn, exploit the context components discussed in Section 2. The finer details of polarity particle distribution in Romanian have shown the relevance of the established differences between reactions to assertions and reactions to polar questions. These data appear to confirm the predictions made by our characterization of responding assertions. The question that arises now is whether further cross-linguistic data will do the same.

In closing, we list the expectations our approach leads us to: (i) We expect partial overlap in the marking of responding assertions sharing relative and/or absolute features. (ii) We expect the possibility of relative polarity markers as well as that of absolute markers. (iii) Given the more marked nature of reversing moves, we do not expect a language with a [same] particle but without a [reverse] one, though the opposite situation is not surprising. (iv) We expect [reverse] to be more marked in denials than in reverse answers, and therefore we would find it surprising if a language were to use a [reverse] particle in reverse answers but not in denials. (v) We expect the [reverse, +] combination to be specially marked relative to [reverse, - ]. We therefore do not expect to find languages that have a special particle used in [reverse, - ] responses but no special marker for $[$ reverse,+$]$ ones. In the same vein, we expect the $[$ same, -$]$ combination to be specially marked relative to $[$ same,+$]$.

\section{Conclusion}

We have focused here on default assertions and default polar questions with the aim of capturing both the similarities and the differences found in a subset of reactions to these speech acts. The details of the context structures we have used, as well as the characterization of the main contextual effects of default assertions and default polar questions allow us to capture both what is the same and what is different in these two

\footnotetext{
${ }^{24}$ We are grateful to undergraduates from UCSC who brought the 'yeah.no' answer to our attention.
} 
speech acts, as well as in conversational moves that confirm/reverse an assertion or a polar question. We thereby have accounted for the partial overlap found in the form of these reactions.

Our discussion, we hope, opens the door toward a systematic cross-linguistic study of responding assertions and the particles that are used in them, a topic that has not received the attention it deserves. On the other hand, there are many much discussed issues that we have been silent about, such as the various types of bias various types of non-default questions and assertions come with. We hope to have nevertheless shown that focusing on the details of the reactions to default polar questions and default assertions turns out to be a useful step in understanding the nature of these two speech acts and the contexts in which they occur.

\section{References}

Asher, Nicholas, \& Lascarides, Alex. 2003. Logics of Conversation. Cambridge: Cambridge University Press.

Asher, Nicholas, \& Lascarides, Alex. 2008. Commitments, beliefs and intentions in dialogue. Pages 35-42 of: Proceedings of the 12th Workshop on the Semantics and Pragmatics of Dialogue (Londial).

Asher, Nicholas, \& Reese, N. 2007. Intonation and Discourse: Biased Questions. Pages 1-38 of: Ishihara, S., Jannedy, S., \& Schwarz, A. (eds), Working Papers of the SFB632, Interdisciplinary Studies on Information Structure (ISIS), vol. 8. Potsdam: Universitatsverlag Potsdam.

Büring, Daniel. 2003. On D-trees, Beans, and Accents. Linguistics and Philosophy, 26, 511-545.

Carlson, L. 1983. Dialogue Games. Dordrecht: Reidel.

Clark, H. 1992. Arenas of Language Use. Chicago: University of Chicago Press.

Clark, H., \& Schaefer, E. F. 1989. Contributing to Discourse. Cognitive Science, 13, 259-294.

Farkas, Donka F. to appear. Polarity Particles in Romanian. In: Di Sciullo, Anna Maria, \& Hill, Virginia (eds), Interface Properties: Edges, Heads and Projections. John Benjamins.

Ginzburg, J. 1995. Resolving Questions, I \& II. Linguistics and Philosophy, 18, 459-527 \& 567-609.

Ginzburg, Jonathan. 1996. Dynamics and the Semantics of Dialogue. In: Seligman, J. (ed), Language, Logic, and Computation, vol. 1. Stanford: CSLI Lecture Notes.

Ginzburg, Jonathan. forthcoming. The Interactive Stance: Meaning for Conversation. Stanford, California: CSLI Publications.

Groenendijk, Jeroen. 2003. Questions and answers: Semantics and logic. Pages 16-23 of: Procedings of the 2nd ColgNET-ElsET Smposium. Questions and Answers: Theoretical and Applied Perspectives.

Gunlogson, Christine. 2001. True to Form: Rising and Falling Declaratives as Questions in English. New York, London: Routledge.

Gunlogson, Christine. 2008. A question of commitment. Belgian Journal of Linguistics, 22, 101-136.

Hamblin, C. I. 1971. Mathematical Models of Dialogue. Theoria, 37, 130-155. 
Horn, Laurence. 2001. A Natural History of Negation. CSLI.

Karttunen, Lauri. 1977. Syntax and Semantics of Questions. Linguistics and Philosophy, 1(1), 3-44.

Kramer, Ruth, \& Rawlins, Kyle. to appear. Polarity particles: an ellipsis account. In: Proceedings of NELS 39.

Krifka, Manfred. 2001. Quantifying into Question Acts. Natural Language Semantics, 9, 1 - 40.

Lascarides, Alex, \& Asher, Nicholas. 2008. Agreement and disputes in dialogue. Pages 29 - 36 of: Proceedings of the 9th SigDial Workshop on Discourse and Dialogue (SIGDIAL.

Merchant, Jason. 2004. Fragments and Ellipsis. Linguistics and Philosophy, 27(6), 661-738.

Poesio, Massimo, \& Traum, David. 1998. Towards an Axiomatization of Dialogue Acts. Pages 207-222 of: Proceedings of the Twente Workshop on the Formal Semantics and Pragmatics of Dialogues (13th Twente Workshop on Language Technology.

Pope, Emily. 1976. Questions and Answers in English. The Hague: Mouton.

Roberts, Craige. 1996. Information structure in discourse. In: Yoon, J. H, \& Kathol, A. (eds), OSU Working Papers in Linguistics 49: Papers in Semantics. Columbus, Ohio: Ohio State University.

Romero, Maribel, \& Han, Chung-hye. 2004. On Negative Yes/No Questions. Linguistics and Philosophy, 27, 609-658.

Rooy, Robert van, \& Safarova, Marie. 2003. On polar questions. In: Young, R., \& Zhou, Y. (eds), Proceedings of SALT XIII. Ithaca, NY, Cornell University.

Sacks, H. 1992. Lectures on Conversation. Oxford, England: Blackwell.

Sadock, J. M., \& Zwicky, A. M. 1985. Speech act distinctions in syntax. Pages 155-196 of: Shopen, T. (ed), Language typology and syntactic description. Cambridge, England: Cambridge University Press.

Sandt, R. van der, \& Maier, E. 2003. Denials in discourse. In: Michigan Philosophy and Linguistics Workshop.

Stalnaker, Robert. 1978. Assertion. Pages 315 -332 of: Cole, P. (ed), Syntax and Semantics: 9. New York: Academic Press.

Walker, Marilyn A. 1996. Inferring acceptance and rejection in dialogue by default rules of inference. Language and Speech, 39(2), 39-2. 